\title{
Research about calculating Beidou dual-frequency combination fixed wide lane ambiguity
}

\author{
Guannan $\mathrm{Xu}^{\mathrm{a}}$, Jie Wang \\ Navigation Engineering, Naval University of Engineering, Wuhan 430033, China \\ a15623532850@163.com
}

Keywords: move relative positioning, LAMBDA method, the ambiguity, double-frequency wide lane.

\begin{abstract}
In view of the key technology of solving ambiguity of high precision move positioning, due to the traditional LAMBDA method to solve ambiguity, the passage improve and put forward a double-frequency observation model calculating ambiguity wide lane. Combined with the observation model and partial data, through self-programming for any four Beidou satellites wide lane ambiguity and satellite ambiguity solving process was simulated. The simulation results show that on the one hand, double-frequency wide lane portfolio model can be effectively applied to the fixed ambiguity, on the other hand, the ambiguity of solving process of the high reliability.
\end{abstract}

\section{Introduction}

For high precision move positioning, real-time solution of ambiguity is a difficulty and a focus research question, is also a key link in the process of decision system positioning precision. The current common methods of OTF generally require multiple epoch observation data, the high precision dynamic real-time meet sex is bad, so we need for the whole week ambiguity study of single epoch algorithm. However, the traditional single epoch ambiguity during the least-squares solution, tend to be less because observation epoch in observation method equation appear rank-defect phenomenon, causes are not present in the observation matrix inverse matrix to solve. According to the above problem, based on the analysis of the traditional method to solve the fuzzy degree, put forward a kind of linear combination based on double-frequency observation model, and through the step-by-step elimination method of solving ambiguity and the baseline vector, respectively.

\section{The establishment of the observation equation}

Traditional LAMBDA method in solving ambiguity, because of the normal equation occurs on observation epoch is less in rank and can't get the observation matrix inverse matrix, is unable to get the ambiguity float solution. On the basis of this method to make improvement, process is as follows:

Now suppose a combination observation model of Beidou frequency point $B_{1}$ and $B_{2}$, remember $N_{\alpha \beta}$. Ambiguity and baseline vector of the observation equation containing two unknowns, for the convenience of solving, now through step-by-step elimination method expunction first one unknown, unknown to solve another stay. Take elimination unknown coordinate first, then determine the method of ambiguity, after elimination unknown coordinate, through the pseudorange and wide lane combination of observation equations can draw wide lane observation the ambiguity float solution of the factor and its covariance matrix, using the LAMBDA method based on covariance factor to determine the ambiguity search space of fixed. Finally, according to the linear relation, calculate $B_{1}$ and $B_{2}$.

In the process of solving $B_{1}$ and $B_{2}$, using the same method to determine the ambiguity step by step. In double-frequency wide lane observations of carrier wave equation after elimination observations of $B_{2}$, the observation equation containing to beg frequency ambiguity parameters $B_{1}$ and three coordinates. In order to reduce the ionosphere effect, use the combination with $B_{1}$ value ambiguity solution as a search criteria, determine $B_{1}$ and $B_{2}$. 
A base station and rover at the same time can be observed by $n$ satellite, for two of the satellite as $i$ and $j$, and in a particular epoch time can get any two stand 1 and 2 about $B_{1}$ wide lane combination observation equation is:

$$
\lambda_{\mathrm{W}} \varphi_{12, W}^{i j}=\rho_{12, W}^{i j}+\lambda_{W} N_{12, W}^{i j}+\varepsilon_{12, W}^{i j}
$$

Among them, $\lambda$ as the carrier wavelength, $\varphi$ as the carrier phase observations, $\rho$ as the distance between the star and the carrier, $N$ as ambiguity, $\varepsilon$ as the carrier phase observation noise, $W$ as wide lane combination.

According to the wide lane combination observation equation, also can give wide lane carrier all ambiguity least squares equation:

$$
\left[\begin{array}{cc}
A^{\mathrm{T}} C^{-1} A & A^{T} C^{-1} \mathrm{~B} \\
\mathrm{~B}^{\mathrm{T}} \mathrm{C}^{-1} \mathrm{~A} & \mathrm{~B}^{\mathrm{T}} \mathrm{C}^{-1} \mathrm{~B}
\end{array}\right]\left[\begin{array}{c}
\mathrm{X} \\
\mathrm{N}_{\alpha \beta}
\end{array}\right]=\left[\begin{array}{l}
\mathrm{A}^{\mathrm{T}} \mathrm{C}^{-1} \mathrm{~L} \\
\mathrm{~B}^{\mathrm{T}} \mathrm{C}^{-1} \mathrm{~L}
\end{array}\right]
$$

On the type, $X$ as the baseline vector, $N_{\alpha \beta}$ as the combination of double difference ambiguity vector, respectively, $A$ and $B$ as coefficient matrix and $C^{-1}$ as weight matrix. According to the coefficient of combination, have established under type:

$$
\varphi_{\alpha \beta}=\alpha \varphi_{\mathrm{B} 1}+\beta \varphi_{\mathrm{B} 2}
$$

After the combination ambiguity, $\mathrm{N}_{\alpha \beta}$ can be determined according to the traditional least squares method, as only a set of coefficient $\alpha$ and $\beta$, the mathematical relationship is unable to correctly solve their ambiguity, so if any search for a new set of coefficients $\eta$ and $\gamma$, as the type of combination, it may solve ambiguity $\mathrm{N}_{1}$ and $\mathrm{N}_{2}$ in accordance with the following combination equation , after solving the ambiguity, also can according to (2) the solution of the baseline vector.

$$
\left[\begin{array}{l}
\mathrm{N}_{\alpha \beta} \\
\mathrm{N}_{\eta \gamma}
\end{array}\right]=\left[\begin{array}{ll}
\alpha & \beta \\
\eta & \gamma
\end{array}\right]\left[\begin{array}{l}
\mathrm{N}_{1} \\
\mathrm{~N}_{2}
\end{array}\right]
$$

\section{The simulation analysis}

For dual-band linear combination model and the method above, the following through self-programming beidou satellite data from the simulation of a receiver for wide lane combination ambiguity solution, among them, the simulation of satellite PRN number 9,16,23,26 respectively, and the simulation results are as follows:

See from figure 1 four satellite simulation results, each satellite of double difference ambiguity wide lane has been properly fixed at about 60 epoch. This can explain, double-frequency composed method can effectively fixed ambiguity.

After solving the combinatorial ambiguity of $N_{\alpha \beta}$ wide lane, according to the above equation (3), can get baseline, thus acquiring station real time coordinates. In the observation equation, the coordinates of unknown parameter contains three station correct number and $N-1$ ambiguity parameter values, a total of $N+2$ parameters, a number of $2(N-1)$ observation equations. The GNSS positioning principle shows that only when four or more than four observable satellite conditions can solve ambiguity. But in general, for the Beidou system, due to the GEO and IGSO satellites is mainly covered in the asia-pacific region, the number of two satellites at any moment can be 7 to 10 , so reach calculating condition. 




C09 satellite

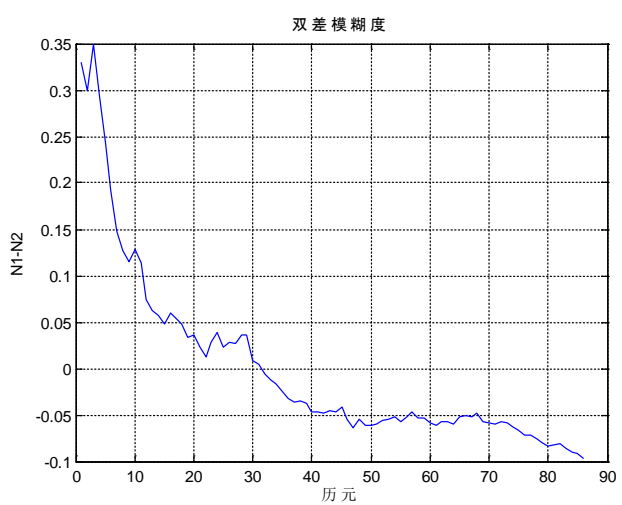

C23 satellite

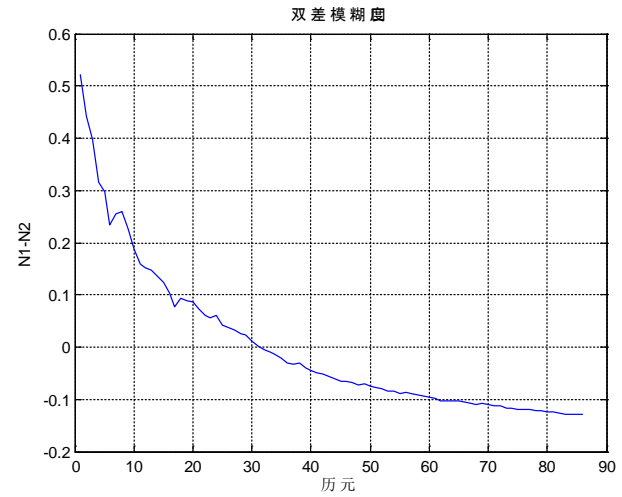

C16 satellite

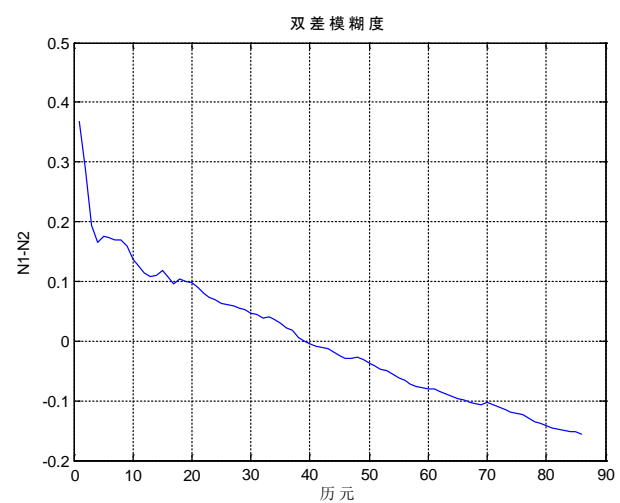

C26 satellite

Figure 1. Simulation results of Beidou satellite of double wide lane ambiguity

In the process of double difference ambiguity fixed after the simulation, to solve their satellite ambiguity of a frequency point, now of the above four satellite simulation, the simulation results are as follows:

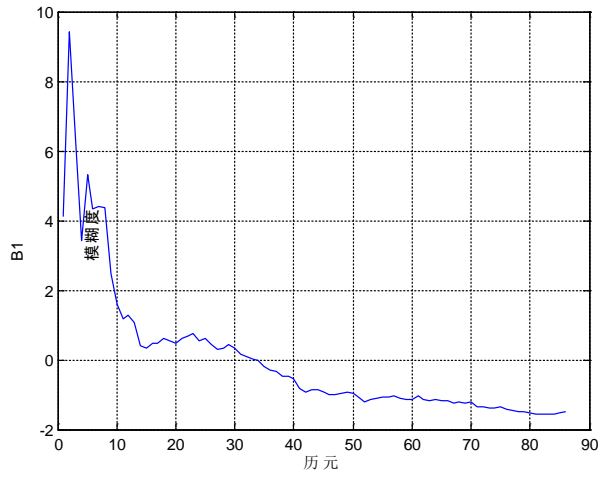

PRN09 B1 ambiguity

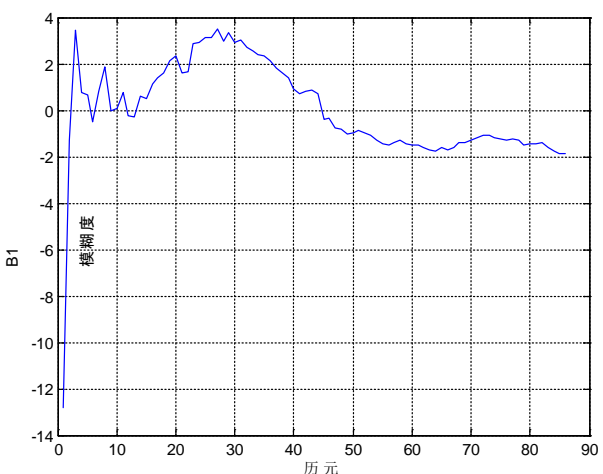

PRN23 B1 ambiguity



PRN16 B1 ambiguity

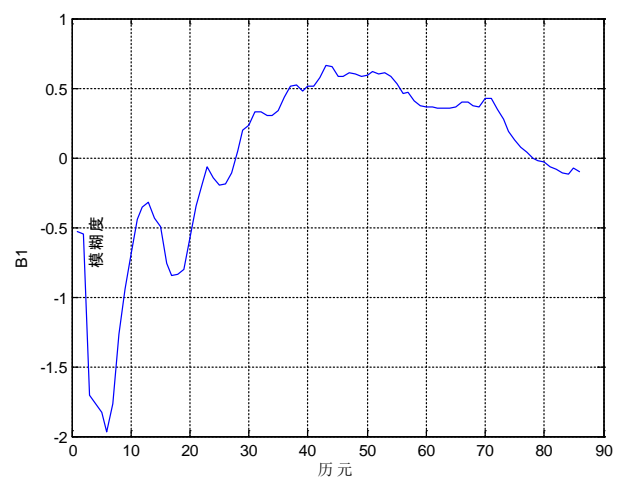

PRN26 B1 ambiguity

Figure 2 .The simulation results of $\nabla \Delta B_{1}$ of Beidou satellites 
Can be seen from the figure 2, the fixed $\nabla \Delta B_{1}$ fluctuates in smaller scale, and fixed time is shorter, it can be seen, the method of fixed $\nabla \Delta B_{1}$ is high reliability.

\section{Conclusion}

This paper puts forward a dynamic relative positioning model of carrier phase double difference. First of all, through the establishment of combination fixed wide lane ambiguity, step-by-step elimination method is used to solve the ambiguity $B_{1}$ of double difference observation, again according to the linear combination relation of solving ambiguity $B_{2}$, then, can get the real-time station coordinates. Through the example simulation results show that the combined model and fixed method of usability is strong. Through analysis and study of this paper, the selection of Beidou observation model and the ambiguity of solving a certain role.

\section{Reference}

[1] Shi Lin. GPS ambiguity and its application in attitude measurement of the whole week research [D]. Wuhan: wuhan university of technology, 2008.04.

[2] li-long liu. Dynamic research on dynamic GPS precise positioning theory and its application [D]. Wuhan: wuhan university, 200501.

[3] guo-rong Yu. The GPS dynamic relative positioning algorithm based on mobile reference station research [D]. Wuhan: wuhan university, 2003.10.

[4] GeMaoRong, Xie Baotong. Dynamic of dynamic GPS real-time differential positioning [J]. Journal of engineering survey, 1998, (4) : 55 to 57.

[5] GeMaoRong, static Jun. GPS relative navigation application in spacecraft rendezvous and docking [J]. Bulletin of surveying and mapping, 1998, (5) : 6-7.

[6] guang-jun liu, Ceng Jibin. GPS dynamic relative navigation for spacecraft rendezvous and docking studies and OTF decoding method [J]. Journal of aircraft measurement and control, 2000, 12 (2) : 86-93.

[7] YangRenGui yun-bin yuan, European JiKun. Phase difference technique is applied to real-time spacecraft rendezvous and docking study [J]. Chinese science: astronomy physics mechanics, 2010, 40 (5) : 651-657.

[8] Luo n. Precise Relative Positioning of Multiple Moving Platforms Using GPS Carrier Phase observables [D]. University of Calgary, 2001.

[9] Hemrann B R, Evans, A to G, Law S C, et al. The Kinematic GPS positioning on the fly relative to A moving reference [J]. Journal of the Institute of Navigation, and 1995 (3) : 487-501.

[10] Jonge P D, Tiberius c. The LAMBDA method for integer ambiguity estimation: implementation aspects [R]. Publications of The Delft Gcodetie Computing Centre LGR - Series, 1996 (12) : 8-16, 20-32. 